\title{
Comparative Study on Electrode Arrangement in Electrokinetic Remediation of Contaminated Soil
}

\author{
Yushan Wan†, Juan Zhai and Anwei Wang
}

School of Environmental \& Safety Engineering, Changzhou University, Jiangsu, China

†Corresponding author: Yushan Wan; wanyushan@cczu.edu.cn

Nat. Env. \& Poll. Tech.

Website: www.neptjournal.com

Received: 08-03-2020

Revised: 14-04-2020

Accepted: 15-06-2020

Key Words:

Soil pollution

Electrokinetic remediation

Cadmium removal

\begin{abstract}
To improve the efficiency of electrokinetic remediation of contaminated soil, by introducing single pollutant cadmium and setting up a self-made device test, electrokinetic remediation technology was applied to artificially simulated contaminated soil to explore the influence of three different two-dimensional electrode arrangements [triangle (2-e-1), rectangle (2-e-2) and hexagon (2-e-3)] on electrokinetic remediation of cadmium contaminated soil in this paper. The cathode of the test device was made of high purity (99.9\%) graphite electrode, and the anode was made of stainless steel electrode. Under the condition that the voltage gradient, electrode material, electrolyte and electrification time of the three groups of tests were consistent, the current change, $\mathrm{pH}$ value, conductivity, total cadmium content and cadmium morphology of the three groups of tests were compared. The experimental results showed that the triangle (2-e-1) group, the current increased slowly during the process of power on, the $\mathrm{pH}$ value of soil was over acid, and the transformation rate of various forms of cadmium contained in the soil after the restoration was low. The current change rate of the rectangular (2-e-2) electrode arrangement was the highest, the soil environment was super alkaline, and the removal rate of cadmium was an average level. The hexagonal electrode arrangement (2-e-3) led a large current drop rate and a weakly alkaline $\mathrm{pH}$ environment, the removal efficiency of cadmium at sampling points (S1 and S2) near the anode was as high as $95.54 \%$, and the comprehensive removal rate of cadmium at each sampling point reached $89.6 \%$, moreover, the pollutant removal was uniform and efficient.
\end{abstract}

\section{INTRODUCTION}

Soil electrokinetic remediation is a technology that can remove heavy metals in the soil to a certain extent by applying a low DC power supply or voltage gradient to an electrode inserted into the ground (Bahemmat et al. 2016). The movement of heavy metals in soil has three mechanisms, namely electrophoresis, electroosmosis and electromigration. When the dissolved charged ions are transferred to the charged layer with opposite polarity through electric current artificially applied by the outside world, the ions migrate to the anode and the cathode and accumulate in the electrode area (Paramkusam et al. 2015, Ha et al. 2014), so that heavy metals in the soil are extracted and removed, which is the working principle of soil electrokinetic remediation technology. Due to the advantages of fewer chemical reagents requirement, no secondary pollution, less disturbance to soil and high remediation efficiency, this technology has become a research hotspot.

In the process of electrokinetic remediation of soil, the setting mode, electric field intensity, electrolyte composition, soil physicochemical properties of electrode materials have an impact on the remediation effect, and among these factors, electrode materials and electrode arrangement have a greater impact on the remediation effect (Yang et al. 2014). In the process of electrodynamic repair, the single arrangement of electrodes will cause polarization, which will slow down the repair efficiency and increase energy consumption. In the aspect of electrode material selection, the electrolyte in the cathode chamber will gradually show strong alkaline state during the reaction process, while the liquid in the anode chamber gradually became strongly acidic. Therefore, to avoid the corrosion of the electrode and to take into account the conductivity and economic cost, the graphite electrode and stainless steel electrode are generally used in the laboratory. The morphological distribution of the heavy metal cadmium in the soil and the removal efficiency of cadmium under different electrode arrangements have been extensively analysed (Fang et al. 2015, Danmaliki et al. 2017, Wang et al. 2018). Turer \& Genc (2005) used extraction method to evaluate the effect of two-dimensional electrode configuration on electric remediation and found that the removal rate of lead, zinc and copper in soil varied with the type of pollution. The total removal efficiency of lead, zinc and copper was $29 \%$ when using a circular electrode arrangement, which was lower than that with a flat electrode 
arrangement. Kim et al. (2012) researched the electrokinetic remediation of rice soil contaminated by various metals using four one-dimensional electrode configurations. Zhai et al. (2018) studied the restoration of saline and alkaline land with a two-dimensional hexagonal arrangement of electrodes and found that the salt transport speed and removal efficiency were improved. Hu et al. (2014) compared the advantages and disadvantages of three electrode arrangements through the soil's final electroosmosis effect and found that when the electrodes were arranged in parallel dislocation, not only the displacement was the largest, but the current reduction rate was large, and the economic benefits were high ( $\mathrm{Hu}$ et al. 2014).

In this experiment, the triangle (2-e-1), rectangle (2e-2) and hexagon (2-e-3) arrangements of the electrode were used to conduct electrochemical remediation of contaminated soil. By comparatively analysing the $\mathrm{pH}$ value, conductivity, current change, total cadmium content and the morphological percentage of cadmium at each sampling point under different electrode arrangement mode, a more reasonable electrode arrangement mode was selected. This study provides a theoretical basis for the further application of electrokinetic remediation technology in soil.

\section{MATERIALS AND METHODS}

\section{Experimental Material}

The soil used in the experiment was collected from farmland near the Science and Education Town of Changzhou
University ( $\left.31^{\circ} 41^{\prime} 7.94^{\prime} \mathrm{N} ; 119^{\circ} 57^{\prime} 1.95^{\prime} \mathrm{E}\right)$. After removing leaves and gravel, the soil collected was air-dried and ground, and then subjected to sieving through a $2 \mathrm{~mm}$ nylon sieve. Table 1 shows the physical and chemical properties of the tested clean soil.

$\mathrm{CdCl}_{2}$ solution of a certain concentration was prepared using $\mathrm{CdCl}_{2} \cdot 2 \cdot 5 \mathrm{H}_{2} \mathrm{O}$, and then the clean collected soil was added into $\mathrm{CdCl}_{2}$ solution (the ratio of $\mathrm{CdCl}_{2}$ solution to the soil was $1: 1$ ), followed by even stirring. The soil after culturing (natural culture at room temperature for 15 days) was naturally dried for nearly a week and then ground through a $2 \mathrm{~mm}$ nylon sieve, resulting in the final contaminated soil sample.

\section{Test Device}

The electrokinetic remediation devices for contaminated soil are shown in Fig. 1-1, 1-2, 1-3, respectively. The devices are made of plexiglass and consist of electrode (cathode and anode) chamber, soil chamber and dc power supply. The device depth is $14 \mathrm{~cm}$, in the middle of which cathode chamber has a radius of $3 \mathrm{~cm}$. Electrodes are arranged in triangle, quadrangle and hexagon patterns. Cylindrical graphite (purity 99.9\%) is used for the cathode, and stainless steel is used for the anode. The diameters of cathode and anode are both $1 \mathrm{~cm}$, and the distance between them is $14.5 \mathrm{~cm}$. The whole device also includes porous partition, non-woven filter cloth, peristaltic pump and copper wire, etc.

Table 1: Physical and chemical properties of the tested clean soil.

\begin{tabular}{|lllll|}
\hline $\mathrm{pH}$ value & $\begin{array}{l}\text { Conductivity } \\
\left(\mu \mathrm{S} \cdot \mathrm{cm}^{-1}\right)\end{array}$ & $\begin{array}{l}\text { Organic matter } \\
\left(\mathrm{g} \cdot \mathrm{kg}^{-1}\right)\end{array}$ & $\begin{array}{l}\text { Moisture content } \\
(\%)\end{array}$ & $\begin{array}{l}\text { Cd content } \\
\left(\mathrm{mg} \cdot \mathrm{kg}^{-1}\right)\end{array}$ \\
\hline 7.53 & 136 & 7.63 & 4.37 & 0.056 \\
\hline
\end{tabular}

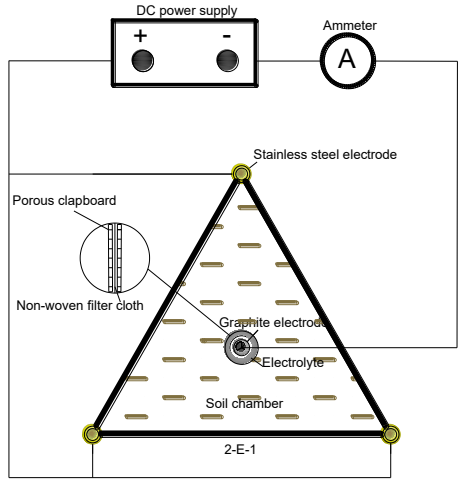

$1-1$

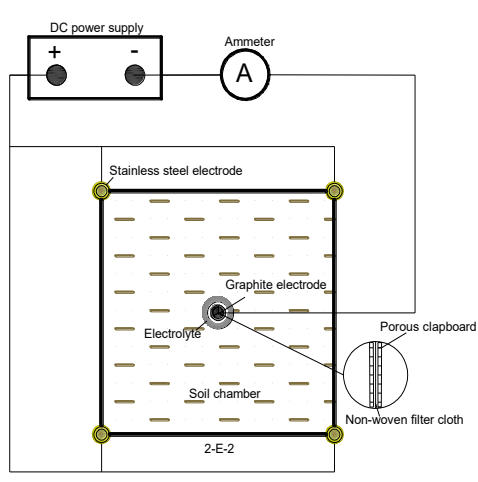

$1-2$

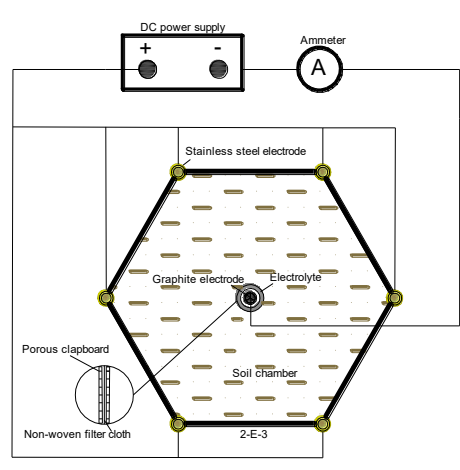

1-3

Fig. 1: Plane schematic diagram of electrode arrangement. 


\section{Test Design and Procedure}

In the soil chamber of the device, $3 \mathrm{~kg}$ of prepared cadmium contaminated soil was placed and compacted with appropriate pressure. In the cathode electrode chamber, the citric acid solution with a concentration of $0.1 \mathrm{~mol} . \mathrm{L}^{-1}$ was added as the cathode electrolyte. A peristaltic pump was used for the circulation of electrolyte to prevent the increase of cathode $\mathrm{pH}$, produce precipitation and inhibit the migration of heavy metals. After static equalization of soil for $24 \mathrm{~h}$, a constant voltage was applied for the test. To better explore the law of Cd migration and change and the optimal electrode arrangement, this experiment adopted the mode of stable and low current output. Since the cathode-anode distances in the three electrode arrangement are all equal to $14.5 \mathrm{~cm}$, so the control voltage gradients are all $2 \mathrm{v} . \mathrm{cm}^{-1}$, and the power-on hours are all set to $120 \mathrm{~h}$. At the same time, equidistant samples were taken from the anode to the cathode in the soil chamber, and 5 sampling points were set, respectively, as shown in Fig. 2. The radius of each sampling point is $1 \mathrm{~cm}$, and the spacing of sampling points is $2.5 \mathrm{~cm}$.

\section{Analytical Methods}

The $\mathrm{pH}$ value and conductivity before and after soil remediation were determined by $\mathrm{pH}$ meter and conductivity meter.
Cadmium in the soil was dissolved by three kinds of acids (nitric acid -perchloric acid-hydrofluoric acid) digestion at high temperature, centrifuged by filter paper, and the precipitated particles in the soil were removed. The filtrate was determined by AAS (flame atomic absorption spectrophotometer). The precipitated particles in the soil were then removed by centrifugation with a filter paper, and the filtrate was then measured by AAS (flame atomic absorption spectrophotometer). Before and after soil remediation, all forms of cadmium in the soil were extracted using the Tessier five-step method, and the same standard curve was used in the test. After the soil samples before and after remediation were digested, the contents of the full amount of cadmium and different forms of cadmium were measured as the same batch (Cameselle 2015).

\section{RESULTS AND DISCUSSION}

At present, studies on electrokinetic remediation of contaminated soil are mostly concentrated on current change after remediation, soil conductivity, soil $\mathrm{pH}$ value, soil total $\mathrm{Cd}$ content and soil $\mathrm{Cd}$ morphological analysis. In this experiment, electrodes were arranged in a two-dimensional arrangement to form a uniform electric field to improve remediation efficiency. Meanwhile, to reflect the spatial
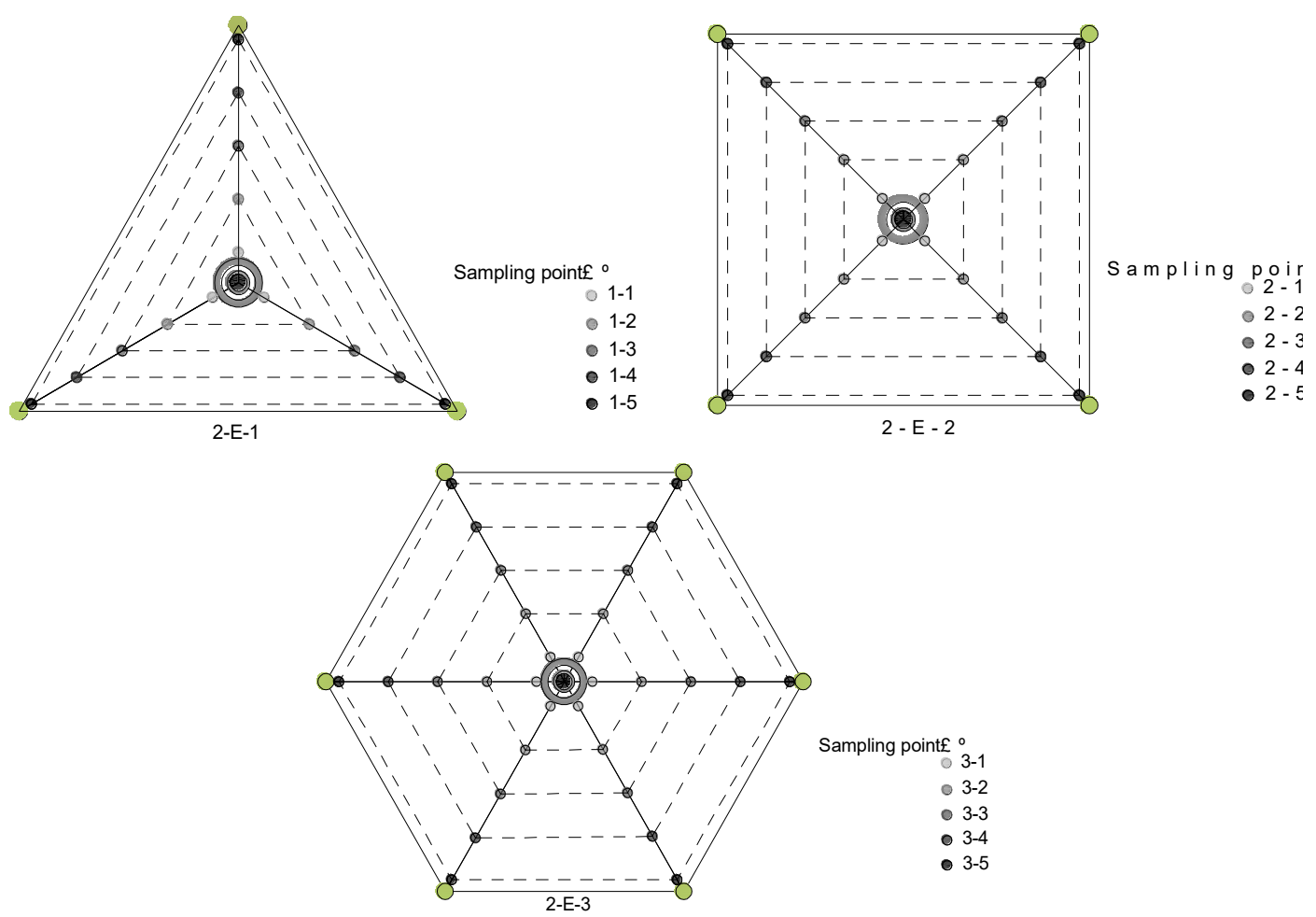

Fig. 2: Schematic diagram of sampling point distribution and electrode position. 
distribution of the repair effect, samples were selected at different geometric positions after remediation. This paper focuses on the analysis of soil $\mathrm{pH}$ value, conductivity, total $\mathrm{Cd}$ content and morphology under the three electrode arrangements, and makes a more comprehensive comparison of the three electrode arrangements in combination with the law of current change.

\section{Current Change During Electrokinetic Remediation}

Under different electrode arrangements, the current changes were recorded every $2 \mathrm{~h}$, and the rule of current changes with reaction time is shown in Fig. 3. It was found that the current changes in the three groups of tests all rose to a peak in a short period and then fell to a steady-state. At the early stage of soil remediation, the current of the three groups of tests increased continuously and reached its peak at $30 \mathrm{~h}$. Due to the hydrolysis between the anode and the cathode, a large amount of $\mathrm{H}^{+}$generated by the anode and $\mathrm{OH}^{-}$generated by the cathode migrate to each other. During the migration of $\mathrm{H}^{+}$ to the cathode, the soil presents an acidification state, which promotes the dissociation of $\mathrm{Cd}^{2+}$ in the soil, increases the migration rate and the concentration of movable ions between soil micropores, so that the current can be gradually increased (Kim et al. 2012). The figure shows that the current increases faster in group 2-E-3 and 2-E-2 than in group 2-E-1. This indicates that more $\mathrm{H}^{+}$is generated by anodic ionization, which is conducive to increasing the migration rate of $\mathrm{Cd}$ ions and further enhancing the removal efficiency of $\mathrm{Cd}$ in the soil. With the increase of soil remediation time, the number of mobile ions between soil micropores and the amount of $\mathrm{Cd}^{2+}$ that can migrate in soil gradually reach a balance. At this point, the nature of the soil itself gradually stabilizes, and the current begins to decline and tends to balance. In addition, the soil can absorb part of $\mathrm{Cd}^{2+}$, which meets $\mathrm{OH}^{-}$ near the cathode, resulting in the formation of precipitation.

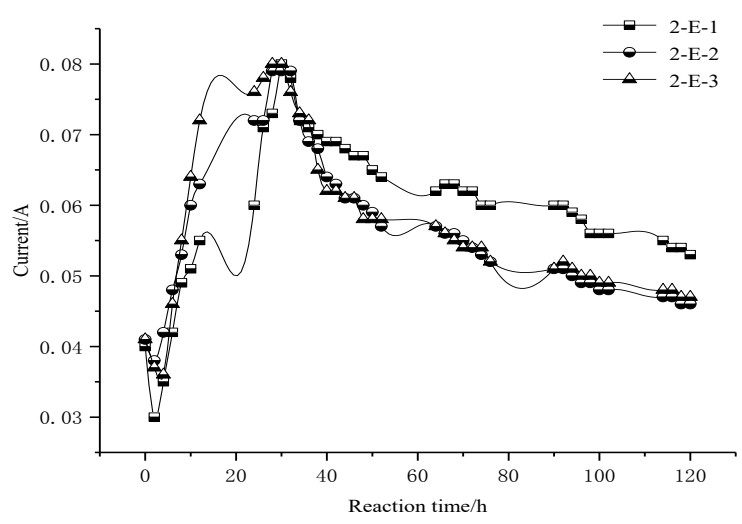

Fig. 3: Current varies with reaction time.
The precipitation may cause the reaction process to stop, which is not conducive to Cd migration.

\section{pH Value at Each Sample Point Changed After Soil Remediation}

Under different electrode arrangements, sampling points were taken from the anode to the cathode at an interval of $2.5 \mathrm{~cm} . \mathrm{pH}$ value at each point was measured after soil remediation, and the mean $\mathrm{pH}$ value was obtained based on multiple sets of parallel samples. The change of $\mathrm{pH}$ value at each sample point in the three test groups was obtained, as shown in Fig. 4. As can be seen from the figure, the $\mathrm{pH}$ value of the clean soil before remediation was 7.53; after $120 \mathrm{~h}$ of electrokinetic remediation, the $\mathrm{pH}$ value of the soil increased with the increase of the distance from the anode. This is because, with the progress of electrolysis reaction in contaminated soil, a large amount of $\mathrm{H}^{+}$generated near the anode and $\mathrm{OH}^{-}$generated near the cathode constantly migrate into the soil, resulting in a continuous decrease or increase of soil $\mathrm{pH}$ near the electrodes at both ends.

From the comparison test, it can be seen that the soil $\mathrm{pH}$ values at sample points $\mathrm{S} 1, \mathrm{~S} 2$ and $\mathrm{S} 3$ in the 2-E-1 group are more acidic than those in the other two groups; while in the 2-E-2 group, the soil $\mathrm{pH}$ value at $\mathrm{S} 5$ is 9.12, which leads to an over alkaline soil environment. Studies have shown that super acidic and super alkaline environments will affect the rate and direction of soil electroosmosis, and reduce the $\mathrm{Cd}$ ion to react with $\mathrm{OH}^{-}$generated by cathode to form precipitation, resulting in blocking of soil micropores, decrease of soil conductivity, and decreased removal efficiency of Cd in the soil (Xu et al. 2016, Amrate et al. 2005). When the pH value of the soil is $3-5$, almost all cadmium in the soil can be extracted and removed. When the $\mathrm{pH}$ value is $6-7$, the extraction efficiency of cadmium decreases to $70 \%$; when the $\mathrm{pH}$ value is greater than 10 , cadmium in the soil cannot

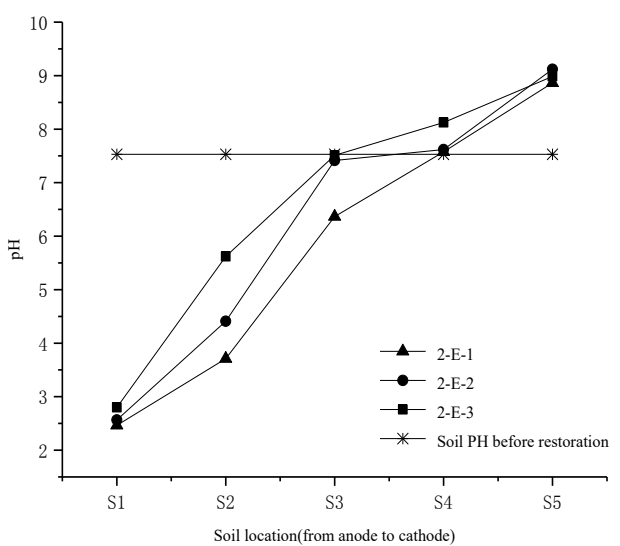

Fig. 4: $\mathrm{pH}$ value of soil at each sampling point after electric remediation. 
be extracted and removed. In the 2-E-3 group test, the $\mathrm{pH}$ value at the sampling point $\mathrm{S} 5$ that is closest to the cathode was 8.98 , and the $\mathrm{pH}$ value at $\mathrm{S} 4$ was 8.13 . Compared with the $\mathrm{pH}$ value of 7.53 before soil remediation, the whole soil reaction chamber presented a weakly alkaline environment, which reduced the adsorption of soil on $\mathrm{Cd}$, promoted the movement of $\mathrm{Cd}$ ions toward the cathode and improved the removal efficiency of $\mathrm{Cd}$.

\section{The Change of Conductivity at Each Sampling Point After Soil Remediation}

Under different electrode arrangements, the conductivity at each sampling point varies with the distance from the anode, as shown in Fig. 5. The conductivity of the tested soil was

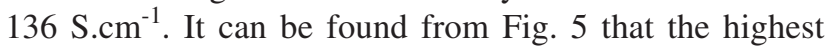
conductivity was at the sampling point (S1) that is closest to the anode, which then began to decline rapidly with a large initial decline rate, and gradually tended to be stable. The conductivity at S5 that is closest to the cathode was the lowest in the whole curve distribution. The conductivity of the soil reflects the degree of difficulty in the movement of ions during electrolysis. In the process of electrokinetic remediation, the lower the soil conductivity is, the less the number of ions that can migrate in the electrolytic reaction is (Adikesavan \& Rajasekar 2018). After the experimental, it can be measured that the electrical conductivity of the S1 sampling point is much higher than that of the initial soil. This is due to that in the electrolysis process, some anions and $\mathrm{Cd}$ ions are enriched near the anode; secondly, soil acidification near the anode can dissolve the precipitates in the soil, resulting in a continuous decrease of $\mathrm{pH}$ value. Different amounts of precipitates dissolved at different sampling points lead to different conductivity values. In the 2-E-3 test group, the conductivity was about twice that of the tested soil, indicating that the arrangement of hexagonal electrodes could increase the migration rate of $\mathrm{Cd}$ ions.

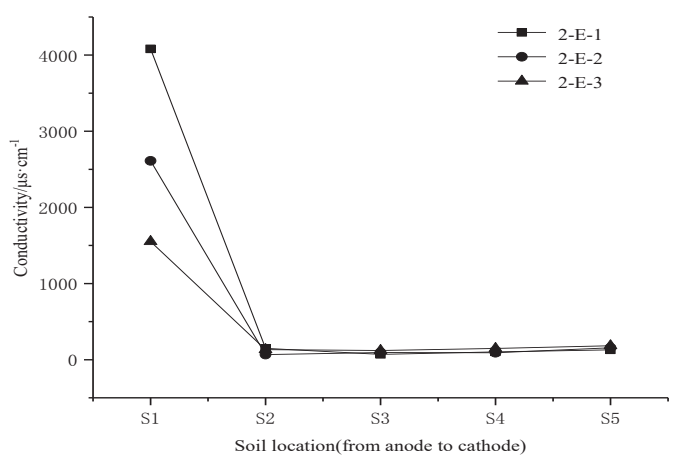

Fig. 5: Soil electrical conductivity at each sampling point after electric remediation.

\section{Total Cadmium Content in Soil After Electrokinetic Remediation}

As can be seen from Fig. 6, after the test, the total cadmium content at all sampling points was lower than that of the tested soil, and the cadmium content gradually increased from the anode to the cathode (S1-S5). Fig. 6 shows that the $\mathrm{Cd}^{2+}$ in the soil migrated from the anode to the cathode, and finally accumulated near the cathode, leading to a concentration change of $\mathrm{Cd}^{2+}$. At the same time, a citric acid solution was used as the circulating electrolyte of the cathode during the electrokinetic remediation process to activate the soil before remediation. Citric acid is an organic acid complexing agent, which has excellent activation and complexation effect on cadmium ions in the tested soil (Bes \& Mench 2008, Habibul et al. 2016). It can reduce the $\mathrm{pH}$ content in the soil, inhibit the adsorption of the soil, and makes the electrokinetic remediation have higher cadmium removal efficiency. The removal efficiency of cadmium at the sampling points near the anode (S1 and S2) was as high as $95.54 \%$, and that at the sampling points away from the cathode and anode (S3 and S4) decreased to about $86.67 \%$. The removal efficiency of cadmium in the area closest to the cathode decreased to $66 \%-72 \%$. The average removal rate of cadmium of the 2-E-3 group was $89.74 \%$, which was increased by $25.63 \%$ compared with that of the 2-E-1 group and 2-E-2 group. The removal rate of cadmium of 2-E-2 group was $12.27 \%$ higher than that of the 2-E-1 group. Therefore, it can be known the electrode arrangement mode in group 2-E-2 and 2-E-3 has a good effect in maintaining the stability of soil properties and removing the uniformity of pollutants.

\section{Morphological Analysis of Cadmium in Soil After Electrokinetic Remediation}

After soil remediation, $\mathrm{pH}$ value, conductivity and $\mathrm{Cd}$ content of the soil all changed. In this study, Tessier five-step method was adopted to conduct a morphological analysis

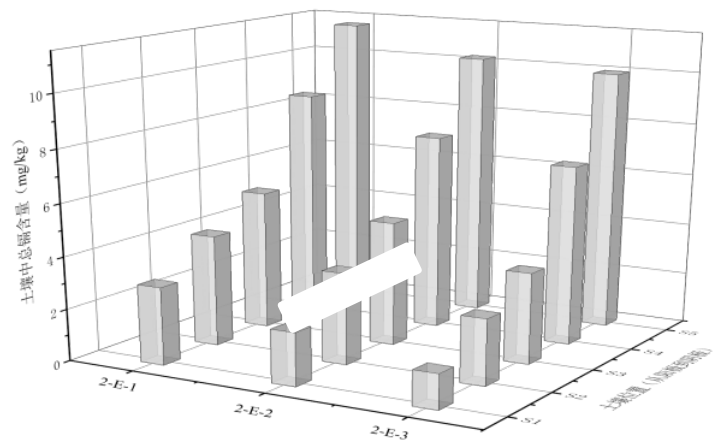

Fig. 6: Total cadmium content at each sampling point after electric repair. 
of cadmium at all sampling points for the three test groups. The percentages of different forms of cadmium are shown in Fig. 7.

As shown in Fig.7, for three test groups of 2-E-1, 2-E-2, 2-E-3, the contents of exchangeable cadmium (EXC) showed an increasing trend, and the contents of $\mathrm{Cd}$ in the form of FeMnOx, Carb and OM all showed a decreasing trend with different decreasing rates, while the content of cadmium in residue state (RES) fluctuated slightly after soil electrokinetic remediation.

In the region (S1-S2) that is close to the cathode, the exchangeable cadmium content is high, and the increased amount after remediation is larger. This is because the soil $\mathrm{pH}$ value in the S1-S2 region is low after electrokinetic remediation, and the cadmium in the unstable state is easy
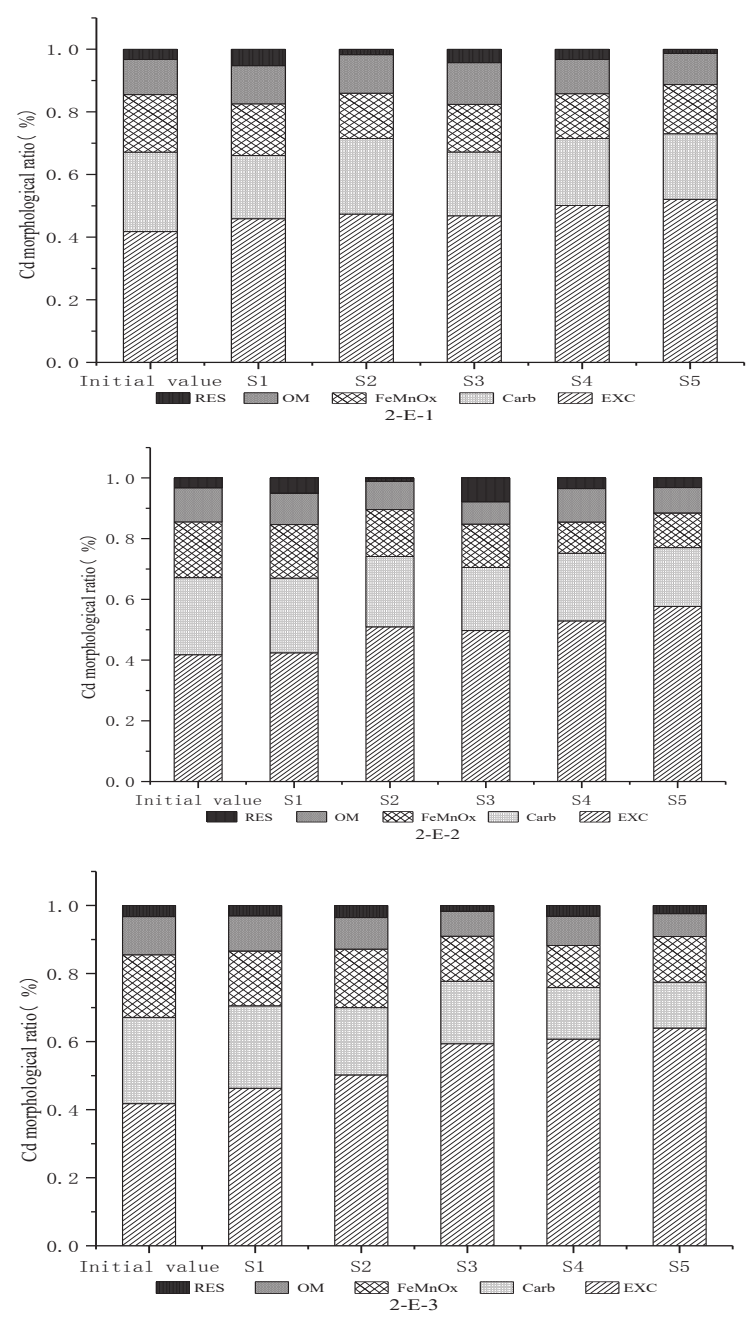

Fig. 7: Morphological distribution of cadmium at various sampling points in soil. to be converted to exchangeable state. The exchangeable cadmium in $\mathrm{S} 1$ of the 2-E-1 group increased from $41.75 \%$ to $45.85 \%$, and that in the 2-E-3 group increased by $4.55 \%$. At the sampling point $\mathrm{S} 2$, the contents of cadmium for three groups increased by $5.60 \%, 8.42 \%$ and $8.44 \%$, respectively. The contents of $\mathrm{Cd}$ in the form of Carb and FeMnOx at the sample point S3-S5 decreased relative to the initial value, and the reduction ratio decreased with the increase of the distance from the anode. This is because under the action of an electric field, partially charged $\mathrm{Cd}^{2+}$ reacts with the $\mathrm{OH}^{-}$generated by the cathode to form precipitation during the migration process, resulting in a smaller current and a lower conversion rate of various forms of cadmium in the contaminated soil (Vengris et al. 2010, Gang et al. 2012). The electrode arrangement for group 2-E-2 and 2-E-3 can increase the electrical conductivity in the soil. In the reductive environment, the cadmium in the form of FeMnOx can be easily converted to the exchangeable cadmium, so the content in the soil is reduced. The content of organic cadmium in soil depends on the content of organic matter in the soil. Through comparing the three test groups, the decline rate of organic cadmium is small and relatively stable, and the organic cadmium region in the 2-E-3 group is evenly distributed, which is conducive to the release of $\mathrm{Cd}$. With the increase of electrokinetic remediation time, the form of cadmium in the soil will gradually change from an unstable state to a stable state.

\section{CONCLUSION}

1. Through the comparison of three two-dimensional electrode arrangements, it was found that the hexagonal electrode arrangement (2-E-3) brought a large current drop rate, a weak alkaline environment. After electrokinetic remediation, the removal efficiency of cadmium at the sampling points ( $\mathrm{S} 1$ and $\mathrm{S} 2$ ) near the anode was as high as $95.54 \%$, and the removal rate of cadmium at each sampling point could be as high as $89.6 \%$. Therefore, it is recommended to adopt a hexagonal arrangement of electrodes when removing Cd pollution in soil by electrokinetic remediation.

2. The Tessier five-step extraction method was adopted to divide the cadmium in soil into five forms after soil remediation. It was found that the cadmium in the form of FeMnOx and Carb was effectively removed. The content of exchangeable $\mathrm{Cd}$ near the cathode area was relatively high, and the content of cadmium in RES state was almost unchanged. Hexagonal electrode arrangement involved an even distribution of organic state $(\mathrm{OM})$ cadmium, which is conducive to the release of $\mathrm{Cd}$. 
3. The practicality of two-dimensional hexagonal electrode arrangement was validated experimentally. With this electrode arrangement, the removal rate of pollutants uniformly is increased, so it has a good application prospect.

\section{ACKNOWLEDGEMENT}

This research was supported by the Postgraduate Research \& Practice Innovation Program of Jiangsu Province, China.

\section{REFERENCES}

Adikesavan, S. and Rajasekar, A. 2018. A statistical approach of zinc remediation using acidophilic bacterium via an integrated approach of bioleaching enhanced electrokinetic remediation (BEER) technology. Chemosphere, 207: 753-763.

Amrate, S., Akretche, D.E., Innocent, C. and Seta, P. 2005. Removal of Pb from a calcareous soil during EDTA-enhanced electrokinetic extraction. Science of the Total Environment, 349: 56-66.

Bahemmat, M., Farahbakhsh, M. and Kianirad, M. 2016. Humic substances-enhanced electroremediation of heavy metals contaminated soil. Journal of Hazardous Materials, 312: 307-318.

Bes, C. and Mench, M. 2008. Remediation of copper-contaminated topsoils from a wood treatment facility using in situ stabilisation. Environmental Pollution, 156: 1128-1138.

Cameselle, C. 2015. Enhancement of electro-osmotic flow during the electrokinetic treatment of a contaminated soil. Electrochimica Acta, 181: 31-38.

Danmaliki, G.I., Saleh, T.A. and Shamsuddeen, A.A. 2017. Response surface methodology optimization of adsorptive desulfurization on nickel/activated carbon. Chemical Engineering Journal, 313: 993-1003.

Fang, L., Fu, R.B. and Xu, Z. 2015. Optimization of electrode configuration in soil electrokinetic remediation. Environmental Science, 36: 678-680.

Gang, L., Guo, S., Li S., Zhang, L. and Wang, S. 2012. Comparison of approaching and fixed anodes for avoiding the 'focusing' effect during electrokinetic remediation of chromium-contaminated soil. Chemical Engineering Journal, 203: 231-238.
Ha, H., Olson, J.R., Bian L. and Rogerson, P.A. 2014. Analysis of heavy metal sources in soil using kriging interpolation on principal components. Environmental Science \& Technology, 48: 4999-5007.

Habibul, N., Hu, Y. and Sheng, G.P. 2016. Microbial fuel cell driving electrokinetic remediation of toxic metal contaminated soils. Journal of Hazardous Materials, 318: 9-14.

Hu, P., Yang, B., Dong, C., Chen, L., Cao, X. and Zhao, J. 2014. Assessment of EDTA heap leaching of an agricultural soil highly contaminated with heavy metals. Chemosphere, 117: 532-537.

Kim, D.H., Jo, S.U., Choi, J.H., Yang, J.S. and Baek, K. 2012. Hexagonal two dimensional electrokinetic systems for restoration of saline agricultural lands: A pilot study. Chemical Engineering Journal, 198: 110-121.

Kim, W.S., Park, G.Y., Kim, D.H., Jung, H.B., Ko, S.H. and Baek, K. 2012. In situ field scale, electrokinetic remediation of multi-metals contaminated paddy soil: Influence of electrode configuration. Electrochimica Acta, 86: 89-95.

Paramkusam, B.R., Srivastava, R.K. and Mohan, D. 2015. Electrokinetic removal of mixed heavy metals from a contaminated low permeable soil by surfactant and chelants. Environmental Earth Sciences, 73: 1191-1204.

Turer, D. and Genc, A. 2005. Assessing effect of electrode configuration on the efficiency of electrokinetic remediation by sequential extraction analysis. Journal of Hazardous Materials, 119: 167-174.

Vengris, T., Binkien , R. and Sveikauskait, A. 2010. Electrokinetic remediation of lead ${ }^{-}$, zinc ${ }^{-}$and cadmium ${ }^{-}$contaminated soil. Journal of Chemical Technology \& Biotechnology, 76: 1165-1170.

Wang, G., Zhang, S., Zhong, Q., Xu, X., Li, T. and Jia, Y. 2018. Effect of soil washing with biodegradable chelators on the toxicity of residual metals and soil biological properties. Science of the Total Environment, 209: 776-782.

Xu, Y., Xu, X., Hou, H., Zhang, J., Zhang, D. and Qian, G. 2016. Moisture content-affected electrokinetic remediation of $\mathrm{Cr}(\mathrm{VI})$-contaminated clay by a hydrocalumite barrier. Environmental Science \& Pollution Research, 23: 6517-6523.

Yang, J.S., Kwon, M.J., Choi, J., Baek, K. and O'Loughlin, E.J. 2014. The transport behavior of $\mathrm{As}, \mathrm{Cu}, \mathrm{Pb}$, and $\mathrm{Zn}$ during electrokinetic remediation of a contaminated soil using electrolyte conditioning. Chemosphere, 117: 79-86.

Zhai, X., Li, Z., Huang, B., Luo, N., Huang, M. and Zhang, Q. 2018. Remediation of multiple heavy metal-contaminated soil through the combination of soil washing and in situ immobilization. Science of the Total Environment, 635: 92-99. 